\title{
Pitavastatin-attenuated cardiac dysfunction in mice with dilated cardiomyopathy via regulation of myocardial calcium handling proteins
}

\author{
WEI HU ${ }^{1, *}$ \\ WEN-BING JIANG ${ }^{2}$ \\ ${ }^{1}$ Department of Intensive Care Unit \\ The First People's Hospital of Hangzhou \\ Nanjing Medical University \\ Hangzhou, 310006 Zhejiang, China \\ 2 Department of Cardiology \\ Wenzhou Third People's Hospital \\ Wenzhou, 325000 Zhejiang, China
}

C57BL/6 mice with dilated cardiomyopathy (DCM) were randomly divided to receive placebo or pitavastatin at a dose of 1 or $3 \mathrm{mg} \mathrm{kg}^{-1} \mathrm{~d}^{-1}$. After 8 weeks treatment, mice with dilated cardiomyopathy developed serious cardiac dysfunction characterized by significantly enhanced left ventricular end-diastolic diameter (LVIDd), decreased left ventricular ejection fraction (LVEF) as well as left ventricular short axis fractional shortening (LVFS), accompanied with enlarged cardiomyocytes, and increased plasma levels of $\mathrm{N}$-terminal pro-B type natriuretic peptide (NT-proBNP) and plasma angiotensin II (AngII) concentration. Moreover, myocardium sarcoplasmic reticulum $\mathrm{Ca}^{2+}$ pump (SERCA-2) activity was decreased. The ratio of phosphorylated phospholamban (PLB) to total PLB decreased significantly with the down-regulation of SERCA-2a and ryanodine receptor (RyR2) expression. Pitavastatin was found to ameliorate the cardiac dysfunction in mice with dilated cardiomyopathy by reversing the changes in the ratios of phosphorylated PLB to total PLB, SERCA-2a and RyR2 via reducing the plasma AngII concentration and the expressions of myocardium angiotensin II type 1 receptor $\left(\mathrm{AT}_{1} \mathrm{R}\right)$ and protein kinase $\mathrm{C}(\mathrm{PKC}) \beta 2$. The possible underlying mechanism might be the regulation of myocardial $\mathrm{AT}_{1} \mathrm{R}-\mathrm{PKC} \beta 2-\mathrm{Ca}^{2+}$ handling proteins.

Keywords: pitavastatin, dilated cardiomyopathy, calcium handling proteins, renin-angiotensin system, protein kinase $C \beta 2$

Dilated cardiomyopathy (DCM) is a multifactorial disease involving cardiac dysfunction and enlargement of one or of both ventricles (1). A number of new anti-heart failure medications, even cytokine therapy (2) or stem cell transplantation (3) have been developed (4) to bring about a glimmer of hope. However, their clinical applications are

* Correspondence; e-mail: huwei19760616@126.com 
W. Hu and W.-B. Jiang: Pitavastatin-attenuated cardiac dysfunction in mice with dilated cardiomyopathy via regulation of myocardial calcium handling proteins, Acta Pharm. 64 (2014) 105-115.

restricted by their high cost and low engraftment. There is still no therapeutic method that effectively reduces the high mortality rate of heart failure except heart transplantation. Therefore, an effective, safe and widely-used agent is urgently needed to attenuate the progress of heart failure.

The 3-hydroxy-3-methyl-glutaryl (HMG)-CoA-reductase inhibitors are the most commonly prescribed agents for hyperlipidemia. In addition to their lipid-lowering action, statins exert a protective effect on the cardiovascular system (5), as indicated by the heart function restoration in ischemic or non-ischemic cardiomyopathy with moderate or severe heart failure (6 ) observed by clinical trials. It has been also reported that statins could ameliorate B type natriuretic peptide (BNP) up-regulation (10) and exert a therapeutic effect in myocarditis and postmyocarditis dilated cardiomyopathy (11). Pitavastatin has been reported to exert a preventive effect on pressure overload-induced heart failure (12) and ischemic heart failure (13). However, in a PEARL study (14), pitavastatin did not reduce cardiac death or hospitalization in patients with left ventricular ejection fraction (LVEF) < $30 \%$. Kjekshus (15) and Chang (16) also reported that statins did not reduce the number of deaths from any cause in patients with systolic heart failure. These controversial outcomes confused us, since little was known about the therapeutic effect and underlying mechanism of pitavastatin on DCM.

The purpose of the current study was to observe the cardiac dysfunction in mice with DCM, to report that pitavastatin could ameliorate the impairment in DCM and to explore the possible molecular mechanism involved.

\section{EXPERIMENTAL}

\section{Animals}

Twenty-four male C57BL/6 mice, aged 8 weeks and weighing approximately $20 \mathrm{~g}$ each, were administered doxorubicin ( $2.5 \mathrm{mg} \mathrm{kg}$, Sigma, USA) intraperitoneally for six days to establish the DCM model (17-9). After 4 weeks, ventricular structure and function were assessed by echocardiography before the animals were randomly divided into three groups, receiving placebo (DCM, $n=8$ ), pitavastatin (powder, Sankyo Company, Japan) at a dose of $1 \mathrm{mg} \mathrm{kg}^{-1} \mathrm{~d}^{-1}(\mathrm{DCM}+\mathrm{LD}, n=8)$ (LD for low dose of pitavastatin) and $3 \mathrm{mg} \mathrm{kg}^{-1} \mathrm{~d}^{-1}$ (DCM+HD, $n=8$ ) (HD for high dose of pitavastatin) respectively, with eight C57BL/ 6 (sham, $n=8$ ) mice without dilated cardiomyopathy as a control. Pitavastatin was administered as a suspension in saline, which was used as a vehicle. The concentration of pitavastatin was $0.1 \mathrm{mg} \mathrm{mL}^{-1}$ and $0.3 \mathrm{mg} \mathrm{mL}^{-1}$ for DCM+LD or DCM+HD, resp. This vehicle was used as a negative control. Pitavastatin and vehicle were administered at a dose of $200 \mu \mathrm{L}$ daily by intragastric gavage for 8 weeks.

All animal experimental procedures were approved by the Animal Care and Use Committee of Nanjing Medical University and performed in accordance with the Guide for the Care and Use of Laboratory Animals (NIH publication No. 85-23, National Academy Press, Washington, DC, USA, revised 1996). 
W. Hu and W.-B. Jiang: Pitavastatin-attenuated cardiac dysfunction in mice with dilated cardiomyopathy via regulation of myocardial calcium handling proteins, Acta Pharm. 64 (2014) 105-115.

\section{Measurement of cardiac function}

Trans-thoracic echocardiographic analysis was performed as previously described (20). M-mode images of the left ventricle were recorded after the animals were anesthetized with isoflurane ( $5 \%$ for induction, $1 \%$ for maintenance). The following parameters were taken to evaluate the cardiac structure and function: left ventricular end-diastolic diameter (LVIDd), left ventricular ejection fraction (LVEF), left ventricular short axis fractional shortening (LVFS). All parameters were assessed using an average of three beats.

\section{Determination of plasma NT-proBNP and angiotensin II concentrations}

Blood samples were collected from the hearts after the animals were euthanized. N-terminal pro-B type natriuretic peptide (NT-proBNP, R \& D Systems, USA) and angiotensin II (AngII ELISA kit from CUSABIO, China) concentrations were determined by ELISA according to the manufacturer's instructions.

\section{Hematoxylin and eosin (HE) staining}

The left ventricle (LV) myocardium was fixed in $4 \%$ formalin, cut transversely, embedded in paraffin, and stained with hematoxylin and eosin. Five randomly selected fields per section were analyzed. Digital photographs were taken using a high-resolution digital image analysis system (QwinV3, Leica, Germany).

\section{Measurement of SERCA-2 activity}

The extracted sarcoplasmic reticulum of cardiac tissues was prepared, and myocardium sarcoplasmic reticulum $\mathrm{Ca}^{2+}$ pump (SERCA-2) activity was measured according to the operating instructions of $\mathrm{Ca}^{2+}$-ATPase kit (Nanjing Jiancheng Bioengineering Institute, China).

\section{Western blot}

Ventricles were removed rapidly from the mice and were stored at $-80^{\circ} \mathrm{C}$. The expressions of angiotensin II type 1 receptor $\left(\mathrm{AT}_{1} \mathrm{R}\right)$, phosphorylated protein kinase $\mathrm{C}$ (phospho-PKC) $\alpha$, phospho-PKC $\beta 1$, phospho-PKC $\beta 2$ (1:300, all from Santa Cruz Biotechnology, Inc., USA), SERCA2a, ryanodine receptor (RyR), phosphorylated phospholamban (phospho-PLB), PLB (1:1000, all from ABCAM, UK) were measured by Western blotting and normalized to the protein level of GAPDH. Total proteins were extracted from the frozen ventricle tissues, and the concentrations were determined using the BCA protein assay. Total $40-\mu$ g protein samples were fractionated by sodium dodecylsulphate-polyacrylamide gel electrophoresis and then transferred to polyvinylidene difluoride membranes. After the membranes were incubated in blocking solution (TBS with $5 \%$ BSA and $0.1 \%$ Tween 20) for 1 hour, primary antibodies were applied to the blots with gentle rocking overnight at $4{ }^{\circ} \mathrm{C}$. Then, the membranes were incubated with horseradish peroxidase-conjugated rabbit or goat secondary antibodies (1:5,000 dilution, Kang Chen Biotechnology, China) for 1 hour at room temperature. The antigen-antibody complexes were detected using an ECL Western-blotting detection reagents with LAS-1000 (FUJIFILM, 
W. Hu and W.-B. Jiang: Pitavastatin-attenuated cardiac dysfunction in mice with dilated cardiomyopathy via regulation of myocardial calcium handling proteins, Acta Pharm. 64 (2014) 105-115.

Japan) detect system. Quantification of immunoreactivity was performed through a densitometric analysis using ImagePro 5.0 (Media Cybernetics, Inc., USA).

\section{Statistical analysis}

Data were presented as mean \pm SEM. Differences between groups were evaluated using the one-way analysis of variance (ANOVA) followed by Fisher's LSD test. Differences were considered statistically significant at $p<0.05$.

\section{RESULTS AND DISCUSSION}

Several measurements were used in the present study, such as echocardiographic, NT-proBNP test and SERCA2 activity, to investigate whether DCM mice developed heart dysfunction. It was found that DCM mice developed systolic heart failure characterized by LVIDd increased by $19.6 \%$ ( $p<0.05)$, and decreased LVEF and LVFS by $39.9(p<0.05)$ and $29.5 \%(p<0.05)$, respectively (Fig. 1). Furthermore, enlarged cardiomyocytes (Fig. 2 ) and increased NT-proBNP (Table I) were observed $(p<0.05)$. In addition, down-regulation of the SERCA2 activity by $26.4 \%(p<0.05)$ was observed (Table II).

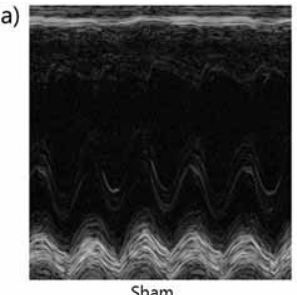

Sham

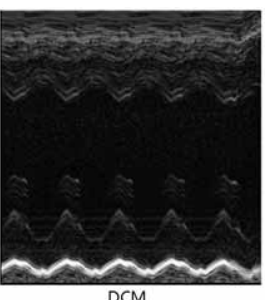

$\mathrm{DCM}$

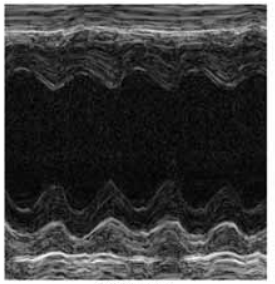

$D C M+L D$

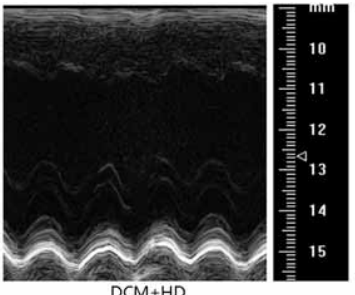

$\mathrm{DCM}+\mathrm{HD}$ b)

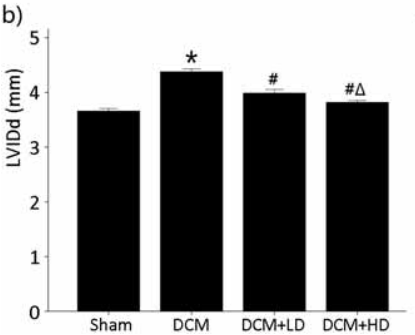

c)

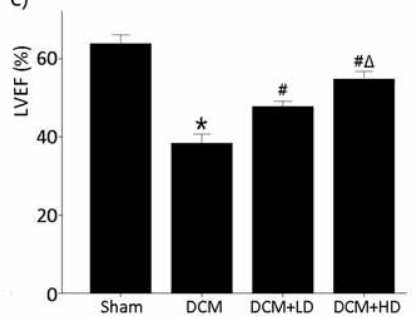

d)

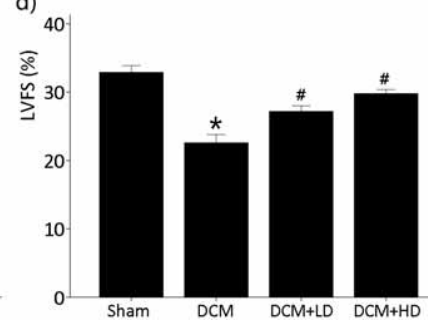

Fig. 1. Echocardiographic data of left ventricular end-diastolic diameter (LVIDd), left ventricular ejection fraction (LVEF) and left ventricular short axis fractional shortening (LVFS). a) M type echocardiographic images of sham, DCM controls, DCM+LD and DCM+HD, b) quantitative analysis of LVIDd in sham, DCM controls, DCM+LD and DCM+HD; c) quantitative analysis of LVEF in sham, DCM controls, DCM+LD and DCM+HD; d) quantitative analysis of LVFS in sham, DCM controls, $\mathrm{DCM}+\mathrm{LD}$ and DCM+HD. Mean $\pm \mathrm{SEM}, n=8 ;{ }^{*} p<0.05$ vs. Sham; ${ }^{*} p<0.05$ vs. DCM controls, ${ }^{\Delta} p<$ 0.05 vs. DCM+LD. 


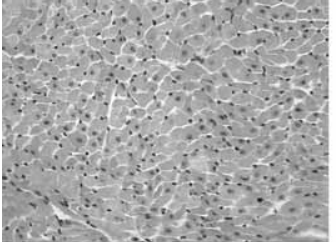

a) Sham
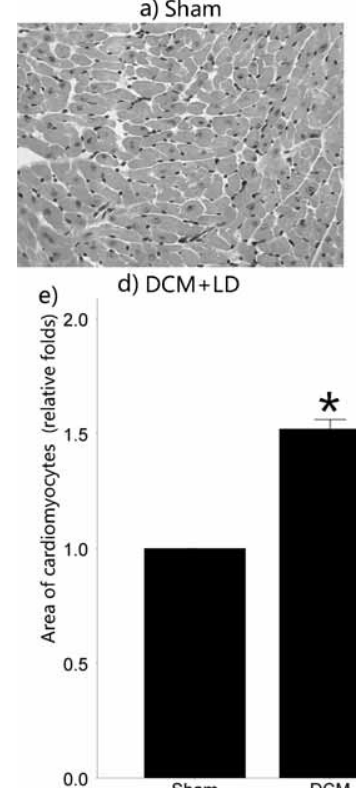

d) $D C M+L D$

Sham

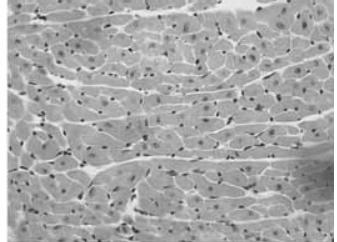

b) DCM

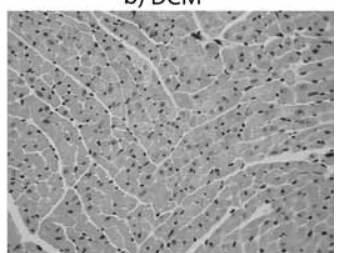

c) $\mathrm{DCM}+\mathrm{HD}$

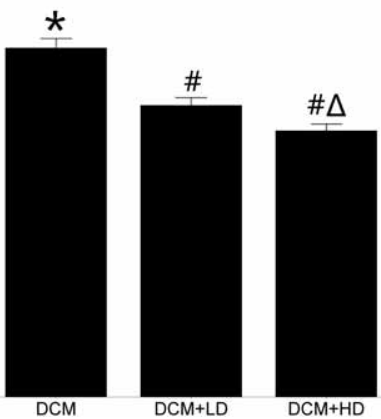

Fig. 2. Hematoxylin and eosin staining. a) Sham control, b) DCM controls, c) DCM+LD, d) $\mathrm{DCM}+\mathrm{HD}$, e) quantitative analysis of the cell size of cardiac myocytes in the four groups and normalized to the sham group. Mean \pm SEM, $n=8 ;{ }^{*} p<0.05$ vs. Sham controls; ${ }^{\#} p<$ 0.05 vs. DCM controls; ${ }^{\Delta} p<0.05$ vs. DCM+LD.

Previous studies have shown that statins can restore heart function in ischemic or non-ischemic cardiomyopathy $(8,11)$, ameliorate BNP up-regulation (10), down-regulate arrhythmic events (6) and reduce sudden cardiac death (21) in addition to their lipid-lowering effects. Our present study also demonstrated that pitavastatin could ameliorate cardiac malfunction in DCM mice, especially in the high dose group $(p<0.05)$ (Figs. 1,2 , Table I). The correlation observed between the doses of pitavastatin and the levels of regression further supported the therapeutic effect of pitavastatin, providing more evidence for pitavastatin as a modulator of pathological changes in dilated cardiomyopathy.

However, there have been controversies over the therapeutic effect of statins on cardiac dysfunction. The UNIVERSE (16) trials reported that statins failed to exert therapeutic effects on the left ventricular remodeling in patients with chronic heart failure. In Kjekshus trial (15), even rosuvastatin could reduce the total number of hospitalizations for heart failure, but produced no effect on heart failure mortality. These confused outcomes might be due to the fact that the subjects with heart failure of ischemic or non-ischemic etiology were not enrolled with a focus on one definite etiology. It is also possible that these subjects had received standard therapies against heart failure, which 
W. Hu and W.-B. Jiang: Pitavastatin-attenuated cardiac dysfunction in mice with dilated cardiomyopathy via regulation of myocardial calcium handling proteins, Acta Pharm. 64 (2014) 105-115.

Table I. Effect of Pitavastatin on plasma NT-proBNP, SERCA-2 activity and angiotensin II level

\begin{tabular}{|c|c|c|c|c|}
\hline Group & Sham & DCM & $\mathrm{DCM}+\mathrm{LD}$ & $\mathrm{DCM}+\mathrm{HD}$ \\
\hline $\begin{array}{l}\text { NT-proBNP concentration } \\
\qquad\left(\mathrm{ng} \mathrm{mL} \mathrm{mL}^{-1}\right)\end{array}$ & $142.80 \pm 22.66$ & $225.70 \pm 19.56^{b}$ & $175.90 \pm 27.48^{c}$ & $162.20 \pm 33.57^{c}$ \\
\hline 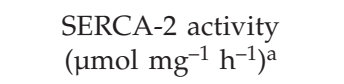 & $6.14 \pm 0.93$ & $4.52 \pm 0.78^{b}$ & $5.71 \pm 1.17^{c}$ & $5.83 \pm 0.33^{c}$ \\
\hline $\begin{array}{l}\text { AngII concentration } \\
\left(\text { ng L L }^{-1}\right)\end{array}$ & $221.00 \pm 16.87$ & $517.20 \pm 26.61^{b}$ & $438.70 \pm 20.74^{\mathrm{c}}$ & $362.80 \pm 26.51^{c}$ \\
\hline
\end{tabular}

NT-proBNP - N-terminal pro-B type natriuretic peptide

SERCA2a - sarcoplasmic reticulum calcium pump.

AngII - Angiotensin II

Mean \pm SEM, $n=8$.

${ }^{a}$ Defined per mg protein; ${ }^{b} p<0.01$ vs. Sham; ${ }^{c} p<0.01$ vs. DCM controls.

Table II. Effect of pitavastatin on SERCA-2 activity

\begin{tabular}{ccccc}
\hline Group & Sham & DCM & DCM+LD & DCM+HD \\
\hline $\begin{array}{c}\text { SERCA-2 activity } \\
\left(\mu \mathrm{mol} \mathrm{mg} \mathrm{m}^{-1} \mathrm{~h}^{-1}\right.\end{array}$ & $6.14 \pm 0.93$ & $4.52 \pm 0.78^{\mathrm{b}}$ & $5.71 \pm 1.17^{\mathrm{c}}$ & $5.83 \pm 0.33^{\mathrm{c}}$ \\
\hline
\end{tabular}

SERCA2a - sarcoplasmic reticulum calcium pump.

a Defined per mg protein. Mean \pm SEM, $n=8$.

b $p<0.01$ vs. Sham; ${ }^{c} p<0.01$ vs. DCM controls.

significantly reduced the mortality of the enrolled subjects before they were treated with statins. Thus, different inclusion and exclusion criteria could cause differences in the pathophysiological and etiological changes observed in heart failure, disturbing the consistence of the results in the cohort. Recently, prospective, randomized, open-label and blinded-endpoint PEARL study (14) of patients with mildly symptomatic heart failure demonstrated that pitavastatin could significantly reduce cardiac death or hospitalization in patients with LVEF $\geq 30 \%$, but not in patients with LVEF $30 \%$. This outcome partly supported our results and provided important data on the role of pitavastatin in the treatment of chronic heart failure.

In order to investigate the mechanism of attenuation of cardiac dysfunction in DCM by pitavastatin, the expressions of myocardium $\mathrm{Ca}^{2+}$-handling proteins were evaluated using Western blot. Our results indicated that the expressions of SERCA2a, RyR2 and the ratio of phosphorylated PLB to total PLB were significantly down-regulated by 41.0 $(p<0.05), 25.2$ and $61.1 \%(p<0.05)$, respectively, in DCM controls compared to those of Sham, which suggested the possible mechanism: that the impaired function of $\mathrm{Ca}^{2+}$ handling proteins could induce $\mathrm{Ca}^{2+}$ overload, causing heart failure. However, these impairments were reversed by pitavastatin treatment, especially in the high dose group $(p<$ 0.05) (Fig. 3). 
W. Hu and W.-B. Jiang: Pitavastatin-attenuated cardiac dysfunction in mice with dilated cardiomyopathy via regulation of myocardial calcium handling proteins, Acta Pharm. 64 (2014) 105-115.
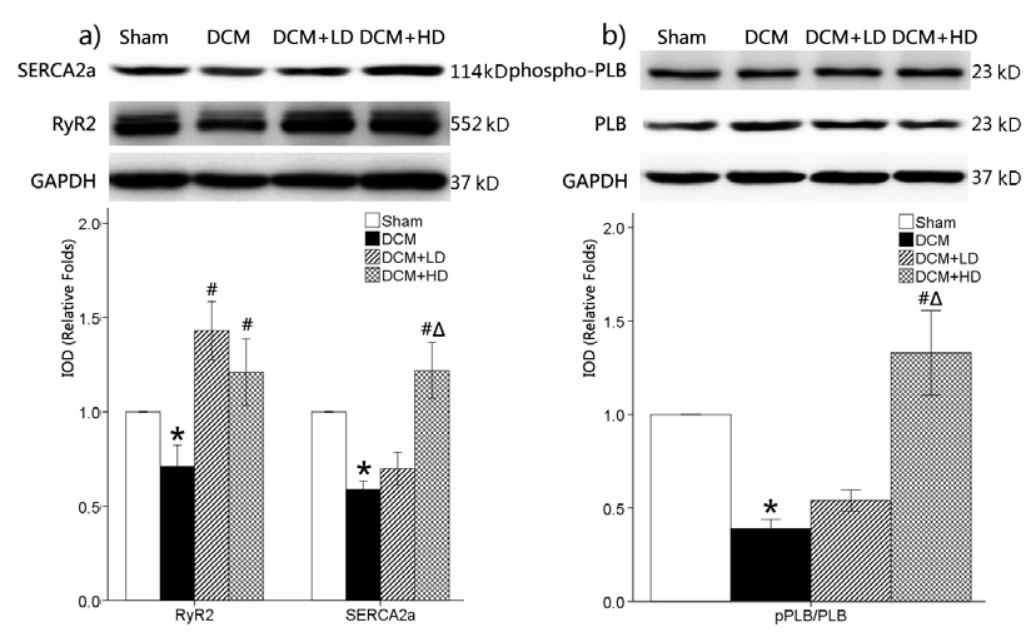

Fig. 3. Expression of $\mathrm{Ca}^{2+}$-handling proteins in myocardium. a) Protein expressions of SERCA2a and RyR2 were measured by Western blot and normalized to the sham group; b) protein expressions of phosphorylated PLB and total PLB were tested, and the ratio of phosphorylated PLB to total PLB was presented and normalized to the sham group (mean \pm SEM, $n=3$ ). ${ }^{*} p<0.05$ vs. Sham; ${ }^{\#} p<0.05$ vs. DCM controls; ${ }^{\Delta} p<0.05$ vs. DCM+LD.

The renin-angiotensin system (RAS) activation has been demonstrated (22) to have an important role in cardiac remodeling and congestive heart failure during the development of DCM. In the present study, it was also found that the concentration of plasma angiotensin II and the expression of $\mathrm{AT}_{1} \mathrm{R}$ were elevated significantly in DCM $(p<0.05)$ (Table I, Fig. 4a). It has been reported that statins could inhibit RAS in type1 diabetes (23, 24) and exert protective effects on hypertensive patients due to RAS suppression (25). We also found that the concentration of AngII and the expression of myocardium $\mathrm{AT}_{1} \mathrm{R}$ were suppressed in the pitavastatin treatment group, which indicated that the circulating and local RAS were involved in the treatment.

Protein kinase C (PKC) signal pathway has been reported to play a crucial role in the $\mathrm{Ca}^{2+}$ regulation (26). Previous studies also reported that RAS inhibition could attenuate cardiac remodeling and heart failure in hypertensive cardiomyopathy $(27,28)$, dilated cardiomyopathy $(29,30)$ and coronary artery disease (31) via inhibition of PKC signaling pathway (26). It was found that the phospho-PКC $\beta 2$, but not phospho-PKC $\alpha$ and phospho-PKC $\beta 1$, was significantly up-regulated by $124.4 \%(p<0.01)$ in DCM, and significantly down-regulated by 30.2 and $50.0 \%(p<0.01)$ in DCM+LD and DCM+HD, respectively (Fig. 4b). Our study has thus provided evidence that pitavastatin can reverse cardiac dysfunction during the development of DCM by interfering with $\mathrm{Ca}^{2+}$ handling proteins via regulating the myocardial RAS-PKC $\beta 2$ signaling pathway in DCM mice.

Our study has also some limitations. The doses of pitavastatin applied to the DCM model were much higher than those in clinical patients. However, the physiological relevance found by the study cannot be neglected even though higher doses are usually administered in animal studies for better therapeutic effects in a short period. Furthermore, 
W. Hu and W.-B. Jiang: Pitavastatin-attenuated cardiac dysfunction in mice with dilated cardiomyopathy via regulation of myocardial calcium handling proteins, Acta Pharm. 64 (2014) 105-115.

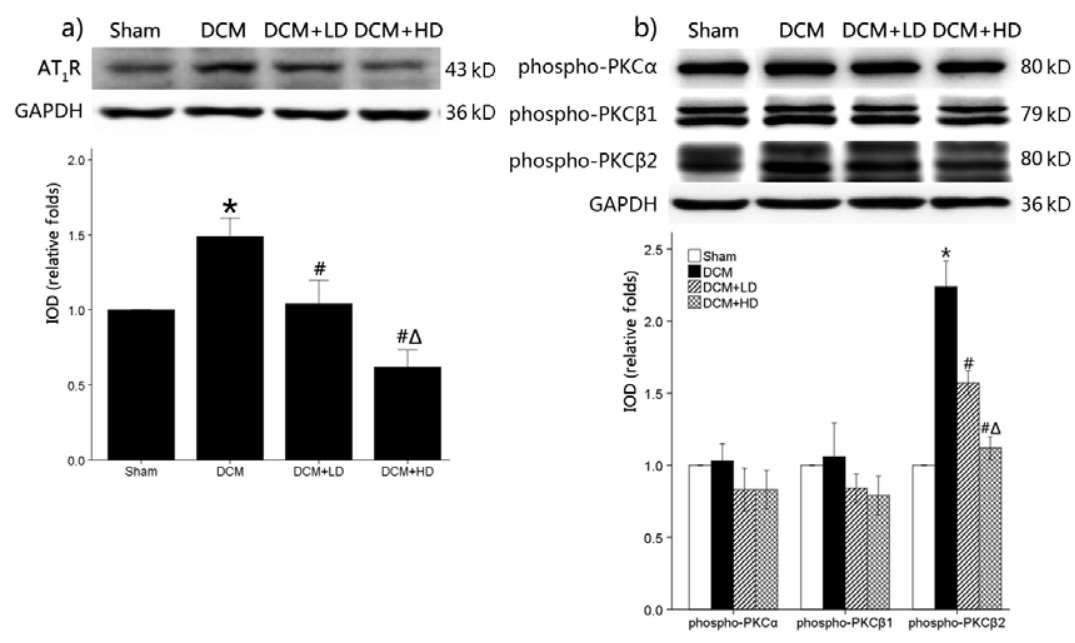

Fig. 4. Protein expression of $\mathrm{AT}_{1} \mathrm{R}, \mathrm{PKC} \alpha, \mathrm{PKC} \beta 1$ and $\mathrm{PKC} \beta 2$ in myocardium: a) protein expressions of $\mathrm{AT}_{1} \mathrm{R}$ was measured by Western blot, and normalized to the sham group; $\mathrm{b}$ ) protein expressions of phospho-PKC $\alpha$, phospho-PKC $\beta 1$ and phospho-PKC $\beta 2$ were tested, and normalized to the sham group (mean \pm SEM, $n=3$ ). ${ }^{*} p<0.05$ vs. Sham; ${ }^{\#} p<0.05$ vs. DCM controls; ${ }^{\Delta} p<0.05$ vs. DCM+LD.

Table III. Effect of pitavastatin on plasma angiotensin II level

\begin{tabular}{ccccc}
\hline Group & Sham & DCM & DCM+LD & DCM+HD \\
\hline AngII concentration $\left(\mathrm{ng} \mathrm{L}^{-1}\right)$ & $221.00 \pm 16.87$ & $517.20 \pm 26.61^{\mathrm{a}}$ & $438.70 \pm 20.74^{\mathrm{b}}$ & $362.80 \pm 26.51^{\mathrm{b}}$ \\
\hline
\end{tabular}

Mean \pm SEM, $n=8$.

${ }^{\mathrm{a}} p<0.01$ vs. Sham, ${ }^{\mathrm{b}} p<0.01$ vs. DCM controls.

pitavastatin was exclusively applied to DCM model. Therefore, its potential therapeutic effect on the DCM may not apply to the patients who have undergone standard anti-heart failure treatments. In addition, our study indicated the possible molecular mechanism of $\mathrm{AT}_{1} \mathrm{R}-\mathrm{PKC} \beta 2-\mathrm{Ca}^{2+}$ handling proteins in the attenuation of heart dysfunction. However, the definitive causal relationship among these three components deserves further investigation.

\section{CONCLUSIONS}

It can be concluded from the present study that pitavastatin exerted a protective effect on dilated cardiomyopathy possibly through down-regulating the circulating and local RAS, followed by inhibition of PKC $\beta 2$ phosphorylation, and consequently promot- 
W. Hu and W.-B. Jiang: Pitavastatin-attenuated cardiac dysfunction in mice with dilated cardiomyopathy via regulation of myocardial calcium handling proteins, Acta Pharm. 64 (2014) 105-115.

ing the phosphorylation of PLB as well as the activity and the expressions of SERCA2a and RyR2, whereby heart function was preserved in the development of DCM.

Acknowledgements. - This work was supported by the Zhejiang Provincial Health Bureau funded projects. We also thank the Sankyo Company for providing pitavastatin.

List of acronyms, abbreviations and symbols. - LVIDd - left ventricular end-diastolic diameter; LVEF - decreased left ventricular ejection fraction; LVFS - left ventricular short axis fractional shortening; NT-proBNP - N-terminal pro-B type natriuretic peptide; AngII - angiotensin II; SERCA-2 - sarcoplasmic reticulum $\mathrm{Ca}^{2+}$ pump; PLB - phospholamban; RyR2 - ryanodine receptor; $\mathrm{AT}_{1} \mathrm{R}$ - angiotensin II type 1 receptor; PKC - protein kinase C; DCM - dilated cardiomyopathy; RAS - renin-angiotensin system; HMG-CoA-reductase - 3-hydroxy-3-methyl-glutaryl-CoA-reductase; LV - left ventricle; $\mathrm{LD}$ - low dose of pitavastatin; HD - high dose of pitavastatin.

\section{REFERENCE}

1. A. Bielecka-Dabrowa, D. P. Mikhailidis, S. Hannam, W. S. Aronow, J. Rysz and M. Banach, Statins and dilated cardiomyopathy: do we have enough data, Expert Opin. Investig. Drugs 20 (2011) 315-323; DOI: 10.1517/13543784.2011.550570.

2. M. Touvron, B. Escoubet, M. Mericskay, A. Angelini, L. Lamotte, M. P. Santini, N. Rosenthal, D. Daegelen, D. Tuil and J. F. Decaux, Locally expressed IGF1 propeptide improves mouse heart function in induced dilated cardiomyopathy by blocking myocardial fibrosis and SRF-dependent CTGF induction, Dis. Model. Mech. 5 (2012) 481-491; DOI: 10.1242/dmm.009456.

3. L. Werner, V. Deutsch, I. Barshack, H. Miller, G. Keren and J. George, Transfer of endothelial progenitor cells improves myocardial performance in rats with dilated cardiomyopathy induced following experimental myocarditis, J. Mol. Cell. Cardiol. 39 (2005) 691-697; DOI: 10.1016/ j.yjmcc.2005.06.015.

4. E. Hoshikawa, Y. Matsumura, T. Kubo, M. Okawa, N. Yamasaki, H. Kitaoka, T. Furuno, J. Takata and Y. L. Doi, Effect of left ventricular reverse remodeling on long-term prognosis after therapy with angiotensin-converting enzyme inhibitors or angiotensin II receptor blockers and beta blockers in patients with idiopathic dilated cardiomyopathy, Am. J. Cardiol. 107 (2011) 1065-1070; DOI: 10.1016/j.amjcard.2010.11.033.

5. W. B. Zhang, Du QJ, H. Li, A. J. Sun, Z. H. Qiu, C. N. Wu, G. Zhao, H. Gong, K. Hu, Y. Z. Zou and J. B. Ge, The therapeutic effect of rosuvastatin on cardiac remodelling from hypertrophy to fibrosis during the end-stage hypertension in rats, J. Cell. Mol. Med. 16 (2012) 2227-2237; DOI: 10.1111/j.1582-4934.2012.01536.x.

6. J. J. Goldberger, H. Subacius, A. Schaechter, A. Howard, R. Berger, A. Shalaby, J. Levine and A. H. Kadish, Effects of statin therapy on arrhythmic events and survival in patients with nonischemic dilated cardiomyopathy, J. Am. Coll. Cardiol. 48 (2006) 1228-1233; DOI: 10.1016/j.jacc. 2006.05.053.

7. F. Tehrani, R. Morrissey, A. Phan, C. Chien and E. R. Schwarz, Statin therapy in patients with diastolic heart failure, Clin. Cardiol. 33 (2010) E1-E5; DOI: 10.1002/clc.20615.

8. S. K. Thambidorai, A. R. Deshmukh, R. W. Walters, P. D. Turner, M. S. Monaghan, A. N. Mooss, C. B. Hunter, D. J. Esterbrooks and S. M. Mohiuddin, Impact of statin use on heart failure mortality, Int. J. Cardiol. 147 (2011) 438-443; DOI: 10.1016/j.ijcard.2010.08.016.

9. T. Sakamoto, S. Kojima, H. Ogawa, H. Shimomura, K. Kimura, Y. Ogata, N. Sakaino and A. Kitagawa, Effects of early statin treatment on symptomatic heart failure and ischemic events after acute myocardial infarction in Japanese, Am. J. Cardiol. 97 (2006) 1165-1171; DOI: 10.1016/ j.amjcard.2005.11.031. 
W. Hu and W.-B. Jiang: Pitavastatin-attenuated cardiac dysfunction in mice with dilated cardiomyopathy via regulation of myocardial calcium handling proteins, Acta Pharm. 64 (2014) 105-115.

10. R. Jarai, C. Kaun, T. W. Weiss, W. S. Speidl, K. Rychli, G. Maurer, K. Huber and J. Wojta, Human cardiac fibroblasts express B-type natriuretic peptide: fluvastatin ameliorates its up-regulation by interleukin-1 alpha, tumour necrosis factor-alpha and transforming growth factor-beta, J. Cell. Mol. Med. 13 (2009) 4415-4421; DOI: 10.1111/j.1582-4934.2009.00704.x.

11. P. E. Lazzerini, P. L. Capecchi and F. Laghi-Pasini, Statins as a new therapeutic perspective in myocarditis and postmyocarditis dilated cardiomyopathy, Cardiovasc. Drugs Ther. 27 (2013) 365-369; DOI: 10.1007/s10557-013-6475-8.

12. Y. Kameda, H. Hasegawa, A. Kubota, H. Tadokoro, Y. Kobayashi, I. Komuro and H. Takano, Effects of pitavastatin on pressure overload-induced heart failure in mice, Circ. J. 76 (2012) 1159-1168; DOI: 10.1253/circj.CJ-11-1114.

13. T. Aoyagi, F. Nakamura, T. Tomaru and T. Toyo-Oka, Beneficial effects of pitavastatin, a 3-hydroxy-3-methylglutaryl coenzyme a reductase inhibitor, on cardiac function in ischemic and nonischemic heart failure, Int. Heart J. 49 (2008) 49-58; DOI: 10.1536/ihj.49.49.

14. H. Takano, H. Mizuma, Y. Kuwabara, Y. Sato, S. Shindo, N. Kotooka, D. Fujimatsu, Y. Kobayashi, T. Inoue, K. Node and I. Komuro, Effects of pitavastatin in Japanese patients with chronic heart failure: the pitavastatin heart failure study (PEARL study), Circ. J. 77 (2013) 917-925; DOI: 10.1253/circj.CJ-12-1062.

15. J. Kjekshus, E. Apetrei, V. Barrios, M. Bohm, J. G. Cleland, J. H. Cornel, P. Dunselman, C. Fonseca, A. Goudev, P. Grande, L. Gullestad, A. Hjalmarson, J. Hradec, A. Janosi, G. Kamensky, M. Komajda, J. Korewicki, T. Kuusi, F. Mach, V. Mareev, J. J. McMurray, N. Ranjith, M. Schaufelberger, J. Vanhaecke, D. J. van Veldhuisen, F. Waagstein, H. Wedel and J. Wikstrand, Rosuvastatin in older patients with systolic heart failure, N. Engl. J. Med. 357 (2007) 2248-2261; DOI: 10.1056/NEJMoa0706201.

16. S. A. Chang, Y. J. Kim, H. W. Lee, D. H. Kim, H. K. Kim, H. J. Chang, D. W. Sohn, B. H. Oh and Y. B. Park, Effect of rosuvastatin on cardiac remodeling, function, and progression to heart failure in hypertensive heart with established left ventricular hypertrophy, Hypertension 54 (2009) 591-597; DOI: 10.1161/HYPERTENSIONAHA.109.131243.

17. K. Krishnamurthy, R. Kanagasabai, L. J. Druhan and G. Ilangovan, Heat shock protein 25-enriched plasma transfusion preconditions the heart against doxorubicin-induced dilated cardiomyopathy in mice, J. Pharmacol. Exp. Ther. 341 (2012) 829-839; DOI: 10.1124/jpet.112.192245.

18. F. N. Gava, E. Zacche, E. M. Ortiz, T. Champion, M. B. Bandarra, R. O. Vasconcelos, J. C. Barbosa and A. A. Camacho, Doxorubicin induced dilated cardiomyopathy in a rabbit model: an update, Res. Vet. Sci. 94 (2013) 115-121; DOI: 10.1016/j.rvsc.2012.07.027.

19. K. Suzuki, B. Murtuza, N. Suzuki, R. T. Smolenski and M. H. Yacoub, Intracoronary infusion of skeletal myoblasts improves cardiac function in doxorubicin-induced heart failure, Circulation 104 (2001) 1213-1217; DOI: 10.1161/hc37t1.094929.

20. M. Sano, T. Minamino, H. Toko, H. Miyauchi, M. Orimo, Y. Qin, H. Akazawa, K. Tateno, Y. Kayama, M. Harada, I. Shimizu, T. Asahara, H. Hamada, S. Tomita, J. D. Molkentin, Y. Zou and I. Komuro, p53-induced inhibition of Hif-1 causes cardiac dysfunction during pressure overload, Nature 446 (2007) 444-448; DOI: 10.1038/nature05602.

21. Y. C. Liao, Y. C. Hsieh, C. Y. Hung, J. L. Huang, C. H. Lin, K. Y. Wang and T. J. Wu, Statin therapy reduces the risk of ventricular arrhythmias, sudden cardiac death, and mortality in heart failure patients: A nationwide population-based cohort study, Int. J. Cardiol. 2013, in press; DOI: 10.1016/j.ijcard.2013.07.036.

22. A. Tidholm, J. Haggstrom and K. Hansson, Effects of dilated cardiomyopathy on the renin-angiotensin-aldosterone system, atrial natriuretic peptide activity, and thyroid hormone concentrations in dogs, Am. J. Vet. Res. 62 (2001) 961-967; DOI: 10.2460/ajvr.2001.62.961.

23. X. Y. Tian, W. T. Wong, A. Xu, Z. Y. Chen, Y. Lu, L. M. Liu, V. W. Lee, C. W. Lau, X. Yao and Y. Huang, Rosuvastatin improves endothelial function in $\mathrm{db} / \mathrm{db}$ mice: role of angiotensin II type 1 receptors and oxidative stress, Br. J. Pharmacol. 164 (2011) 598-606; DOI: 10.1111/j.1476-5381. 2011.01416.x. 
24. H. Toba, T. Mitani, T. Takahashi, N. Imai, R. Serizawa, J. Wang, M. Kobara and T. Nakata, Inhibition of the renal renin-angiotensin system and renoprotection by pitavastatin in type1 diabetes, Clin. Exp. Pharmacol. Physiol. 37 (2010) 1064-1070; DOI: 10.1111/j.1440-1681.2010.05436.x.

25. G. Nickenig and D. G. Harrison, The AT(1)-type angiotensin receptor in oxidative stress and atherogenesis: part I: oxidative stress and atherogenesis, Circulation 105 (2002) 393-396; DOI: 10.1161/hc0302.102618.

26. S. L. Belmonte, B. C. Blaxall, PKC-ing is believing: targeting protein kinase $\mathrm{C}$ in heart failure, Circ. Res. 109 (2011) 1320-1322; DOI: 10.1161/CIRCRESAHA.111.259358.

27. A. L. Beck, M. E. Otto, L. B. D'Avila, F. M. Netto, M. K. Armendaris and A. C. Sposito, Diastolic function parameters are improved by the addition of simvastatin to enalapril-based treatment in hypertensive individuals, Atherosclerosis 222 (2012) 444-448; DOI: 10.1016/j.atherosclerosis. 2012.03.030.

28. X. Zhou, D. Shang, T. Zhang, L. Li, T. Zhou and W. Lu, Modeling of angiotensin II-angiotensin-(1) counterbalance in disease progression in spontaneously hypertensive rats treated with/ without perindopril, Pharmacol. Res. 66 (2012) 177-184; DOI: 10.1016/j.phrs.2012.04.001.

29. M. J. Crespo, N. Cruz, P. I. Altieri and N. Escobales, Enalapril and losartan are more effective than carvedilol in preventing dilated cardiomyopathy in the Syrian cardiomyopathic hamster, J. Cardiovasc. Pharmacol. Ther. 13 (2008) 199-206; DOI: 10.1177/1074248408320006.

30. M. R. O'Grady, M. L. O'Sullivan, S. L. Minors and R. Horne, Efficacy of benazepril hydrochloride to delay the progression of occult dilated cardiomyopathy in Doberman Pinschers, J. Vet. Intern. Med. 23 (2009) 977-983; DOI: 10.1111/j.1939-1676.2009.0346.x.

31. G. Pela, P. Pattoneri, M. Passera, G. Tirabassi, C. Reverberi, A. Montanari and T. Gherli, Long-term effects of perindopril on left ventricular structure and function in patients with stable coronary artery disease: a conventional and Doppler tissue echocardiographic pilot study, J. Cardiovasc. Med. (Hagerstown) 10 (2009) 781-786; DOI: 10.2459/JCM.0b013e32832dbfe9. 